\title{
X-ray-activated long persistent phosphors featuring strong UVC afterglow emissions
}

\author{
Yan-Min Yang ${ }^{1}$, Zhi-Yong Li ${ }^{2}$, Jun-Ying Zhang ${ }^{3}$, Yue Lu ${ }^{3}$, Shao-Qiang Guo ${ }^{3}$, Qing Zhao ${ }^{4}$, Xin Wang ${ }^{1}$, Zi-Jun Yong ${ }^{2}$, \\ Hong Li ${ }^{2}$, Ju-Ping $\mathrm{Ma}^{2}$, Yoshihiro Kuroiwa ${ }^{4}$, Chikako Moriyoshi', Li-Li Hu${ }^{5}$, Li-Yan Zhang ${ }^{5}$, Li-Rong Zheng ${ }^{6}$ and \\ Hong-Tao Sun ${ }^{2}$
}

\begin{abstract}
Phosphors emitting visible and near-infrared persistent luminescence have been explored extensively owing to their unusual properties and commercial interest in their applications such as glow-in-the-dark paints, optical information storage, and in vivo bioimaging. However, no persistent phosphor that features emissions in the ultraviolet $C$ range $(200-280 \mathrm{~nm})$ has been known to exist so far. Here, we demonstrate a strategy for creating a new generation of persistent phosphor that exhibits strong ultraviolet $\mathrm{C}$ emission with an initial power density over 10 milliwatts per square meter and an afterglow of more than $2 \mathrm{~h}$. Experimental characterizations coupled with first-principles calculations have revealed that structural defects associated with oxygen introduction-induced anion vacancies in fluoride elpasolite can function as electron traps, which capture and store a large number of electrons triggered by Xray irradiation. Notably, we show that the ultraviolet $C$ afterglow intensity of the yielded phosphor is sufficiently strong for sterilization. Our discovery of this ultraviolet $C$ afterglow opens up new avenues for research on persistent phosphors, and it offers new perspectives on their applications in terms of sterilization, disinfection, drug release, cancer treatment, anti-counterfeiting, and beyond.
\end{abstract}

\section{Introduction}

Persistent luminescence is an optical phenomenon in which a material stores excitation energy in excited states and the resulting luminescence lasts for an appreciable time after the excitation has stopped ${ }^{1,2}$. Phosphors exhibiting persistent luminescence have received significant attention and have been commercialized for a wide range of applications ${ }^{3-12}$. Since the pioneering work of Matsuzawa et al. ${ }^{13}$ blue persistent $\mathrm{CaAl}_{2} \mathrm{O}_{4}: \mathrm{Eu}^{2+}, \mathrm{Nd}^{3+}$ (ref. ${ }^{14}$ ), green persistent $\mathrm{SrAl}_{2} \mathrm{O}_{4}: \mathrm{Eu}^{2+}, \mathrm{Dy}^{3+}$ (ref. ${ }^{13}$ ), and red persistent $\mathrm{Y}_{2} \mathrm{O}_{2} \mathrm{~S}: \mathrm{Eu}^{3+}, \mathrm{Mg}^{2+}$, and $\mathrm{Ti}^{4+}$ (ref. ${ }^{15}$ ) have been developed. Recent efforts have resulted in the creation of a series of near-infrared (NIR) persistent phosphors, such as

Correspondence: H.-T. Sun (timothyhsun@gmail.com)

${ }^{1}$ College of Physics Science and Technology, Hebei University, 071002 Baoding, China

${ }^{2}$ College of Chemistry, Chemical Engineering and Materials Science, Soochow University, 215123 Jiangsu, China

Full list of author information is available at the end of the article.

These authors contributed equally: Yan-Min Yang, Zhi-Yong Li, Jun-Ying Zhang
$\mathrm{Cr}^{3+}$-doped zinc gallogermanates that can be employed for the in vivo biological imaging and the in vitro targeting of cancerous cells ${ }^{16-26}$. Despite these significant achievements, most of the persistent phosphors reported thus far luminesce in the visible and NIR spectral regions ${ }^{27}$, and no persistent phosphors exhibiting ultraviolet C (UVC) luminescence are known to exist. It is well known that UVC light in the $200-280 \mathrm{~nm}$ range is germicidal, i.e., it can kill bacteria, viruses, and other pathogens by destroying nucleic acids and disabling their ability to multiply ${ }^{28}$. However, the design and synthesis of such a long-lasting phosphor has not been possible thus far.

Two classes of centers, emitters and traps, are required in persistent phosphors. Emitters are optical centers that luminesce after being excited, whereas traps, which are usually associated with defects in host lattices, store excitation energy and then release it slowly to the emitters by virtue of thermal stimulations ${ }^{2}$. Since the emitter determines the emission wavelength, the first required

\section{(c) The Author(s) 2018}

(c) (i) Open Access This article is licensed under a Creative Commons Attribution 4.0 International License, which permits use, sharing, adaptation, distribution and reproduction cc) in any medium or format, as long as you give appropriate credit to the original author(s) and the source, provide a link to the Creative Commons license, and indicate if changes were made. The images or other third party material in this article are included in the article's Creative Commons license, unless indicated otherwise in a credit line to the material. If material is not included in the article's Creative Commons license and your intended use is not permitted by statutory regulation or exceeds the permitted use, you will need to obtain permission directly from the copyright holder. To view a copy of this license, visit http://creativecommons.org/licenses/by/4.0/. 
condition for the design of UVC-persistent phosphors is the choice of a suitable emitter that can luminesce in the UVC. Rare earth (RE) ions have been widely adopted as emitters in persistent phosphors owing to their intraconfigurational transitions. It is noted that some RE ions (e.g., $\mathrm{Pr}^{3+}$ ) possess strong, high-energy, interconfigurational transitions, which thus could be employed as candidate emitters in UVC persistent phosphors ${ }^{29-31}$. The second required condition is the selection of the candidate host materials with a large bandgap and appropriate traps. In this respect, fluoride crystalline lattices can be considered because of their relatively large band gaps and the easy creation of anionic defects ${ }^{32}$. The third condition is that the material system can be efficiently activated (i.e., charged) by suitable excitation sources. Given the large bandgap required for the host, X-ray irradiation may be appropriate. Another requirement relates to the persistent time and intensity of the UVC emission, which should be as long as possible and strong enough to satisfy some practical applications.

Here, we demonstrate a strategy for the creation of a new generation of persistent phosphor featuring UVC emission (which we refer to as a UVC persistent phosphor) by judiciously selecting defect-bearing fluoride elpasolite (i.e., $\mathrm{Cs}_{2} \mathrm{NaYF}_{6}$ ) as a host and $\mathrm{Pr}^{3+}$ ions as emitters. The resulting phosphors show bright UVC emissions that can last over $2 \mathrm{~h}$ after X-ray irradiation. To our knowledge, our work is the first discovery of persistent phosphors capable of luminescing in the UVC. A broad range of experimental characterizations combined with first-principles calculations suggest that oxygen introduction-induced fluorine vacancies act as electron traps, enabling the system to capture a large number of electrons upon X-ray irradiation. This results in strong UVC persistent luminescence corresponding to the $4 f 5 d-4 f^{2}$ transition of $\operatorname{Pr}^{3+}$ when releasing trapped electrons. We show that the UVC persistent luminescence of this phosphor is strong enough to be used for sterilization. Importantly, the phosphors can be charged repeatedly by $\mathrm{X}$-ray or poststimulated by NIR light in the first biological window, thus offering an attractive prospect for applications, such as the in vivo killing of pathogens and cancer cells. This work offers a protocol for the design and preparation of UVC persistent phosphors and opens up new avenues for a wide variety of practical applications.

\section{Results}

We chose elpasolite $\mathrm{Cs}_{2} \mathrm{NaYF}_{6}$ as the host and $\mathrm{Pr}^{3+}$ ions as emitters with the ideas that (1) the Cs element has a strong X-ray absorption capability that makes the system capable of charging by $\mathrm{X}$-ray irradiation, (2) $\mathrm{Cs}_{2} \mathrm{NaYF}_{6}$ has a large bandgap and a propensity for the formation of structural defects that are likely to act as electron traps ${ }^{32}$, and (3) the $4 f 5 d-4 f^{2}$ transition of $\operatorname{Pr}^{3+}$ ions can result in
UVC emission $^{29-31}$. The micrometer-sized phosphor with a nominal composition of $\mathrm{Cs}_{2} \mathrm{NaY}_{(1-x)} \mathrm{F}_{6}: x \mathrm{Pr}^{3+}$ was synthesized through a solid-state reaction method (Supplementary Fig. 1). The major reflections of X-ray diffraction (XRD) patterns for the products can be indexed with an Fm-3m space group that corresponds to the cubic elpasolite (Supplementary Fig. 2), consistent with JCPDS no. 74-0043. In this double perovskite structure, both $\mathrm{Y}$ and $\mathrm{Na}$ coordinate with six fluorine atoms, and doped $\operatorname{Pr}^{3+}$ ions are expected to substitute for $\mathrm{Y}^{3+}$ ions (Supplementary Fig. 3). We first characterized the photoluminescence properties of the yielded product. Under $288 \mathrm{~nm}$ excitation, the product shows luminescence bands at 486 and $610 \mathrm{~nm}$, which can be assigned to the ${ }^{3} \mathrm{P}_{0} \rightarrow{ }^{3} \mathrm{H}_{4}$ and ${ }^{3} \mathrm{P}_{0} \rightarrow{ }^{3} \mathrm{H}_{6}$ transitions of $\mathrm{Pr}^{3+}$, respectively $^{33}$ (Supplementary Fig. 4). We note that after photoexcitation ceases, the photoluminescence quickly disappears, indicating that excitation at $288 \mathrm{~nm}$ cannot result in the charging of this system, which is a necessity for long persistent luminescence. Interestingly, we find that after $\mathrm{X}$-ray irradiation, the sample displayed strong, longlasting UVC persistent luminescence peaking at $\sim 250 \mathrm{~nm}$ owing to the $4 f 5 d \rightarrow{ }^{3} \mathrm{H}_{4}$ electronic transition of $\operatorname{Pr}^{3+}$, accompanied by visible bands of $4 f^{2}-4 f^{2}$ intraconfigurational transitions (Fig. 1a). Figure $1 \mathrm{~b}$ displays the afterglow decay of $\mathrm{Cs}_{2} \mathrm{NaY}_{0.99} \mathrm{~F}_{6}: 0.01 \mathrm{Pr}^{3+}$ detected at $250 \mathrm{~nm}$ following irradiation with an X-ray source for $1000 \mathrm{~s}$ that corresponds to a dose of 20 Gy (Supplementary Fig. 5). We note that all data regarding the afterglow intensity versus time were taken using a spectrofluorometer from 5 min after stopping the X-ray irradiation, to avoid the measurement artifact owing to fast early decay. Clearly, the persistent luminescence of $\mathrm{Cs}_{2} \mathrm{NaY}_{0.99} \mathrm{~F}_{6}: 0.01 \mathrm{Pr}^{3+}$ can last over $2 \mathrm{~h}$, and after $2 \mathrm{~h}$, the intensity is still over one order of magnitude stronger than the background signal of the detection system (Fig. 1b). Additionally, we find that the afterglow behavior is intimately associated with the concentration of $\operatorname{Pr}^{3+}$ ions and the X-ray irradiation duration. The optimal concentration is determined to be $x=0.01$ (Supplementary Fig. 6), and $1000 \mathrm{~s}$ of X-ray irradiation of $\mathrm{Cs}_{2} \mathrm{NaY}_{0.99} \mathrm{~F}_{6}: 0.01 \mathrm{Pr}^{3+}$ gives rise to the strongest UVC persistent luminescence (Supplementary Fig. 7). The afterglow spectra recorded at different times reveal that the lineshape of the luminescence changes with times; the UVC emission decays faster than the visible emissions (Fig. 1b, Supplementary Fig. 8). In addition to the emission bands mentioned above, we note that an emission shoulder at $\sim 340 \mathrm{~nm}$ in the afterglow spectra occurs, which is confirmed to be from the host material (Supplementary Fig. 9).

We further confirmed the UVC emission by using a UVC imager. Figure 1c and Supplementary Fig. 10a display the dependence of the UVC emission intensity on the decay time for $\mathrm{Cs}_{2} \mathrm{NaY}_{0.99} \mathrm{~F}_{6}: 0.01 \mathrm{Pr}^{3+}$ irradiated by the 
$\mathrm{X}$-ray for $1000 \mathrm{~s}$. Clearly, the decay decreases much faster during the first several minutes, and then it occurs slowly (Supplementary Fig. 10b). The initial afterglow UVC emission intensity of $\mathrm{Cs}_{2} \mathrm{NaY}_{0.99} \mathrm{~F}_{6}: 0.01 \mathrm{Pr}^{3+}$ after stopping the $\mathrm{X}$-ray irradiation was estimated to be over 10 milliwatts per square meter (Materials and methods). Heating the 24-h-decayed phosphor at $200^{\circ} \mathrm{C}$ gave rise to strong UVC luminescence again, with a lasting time of over 5 min (Fig. 1d, Supplementary Fig. 10c). Interestingly, we find that these trapped electrons can also be liberated by laser irradiation with various photon energies (Fig. 1e-g, Supplementary Fig. 11). Specifically, irradiation of $450 \mathrm{~nm}$ light causes stronger emissions than those of 730 and $793 \mathrm{~nm}$ light, suggesting that there are more residual trapped electrons located in deeper traps. Collectively, these observations provide an indication that the UVC persistent phosphors developed here feature a diversity of traps, and that a large number of residual electrons is located in deep traps after the room temperature release of electrons in shallow ones. We underscore that this characteristic is extremely attractive and of vital importance for some applications of these UVC phosphors. For instance, although a relatively long-time X-ray irradiation is required to charge the system at present, the release of a

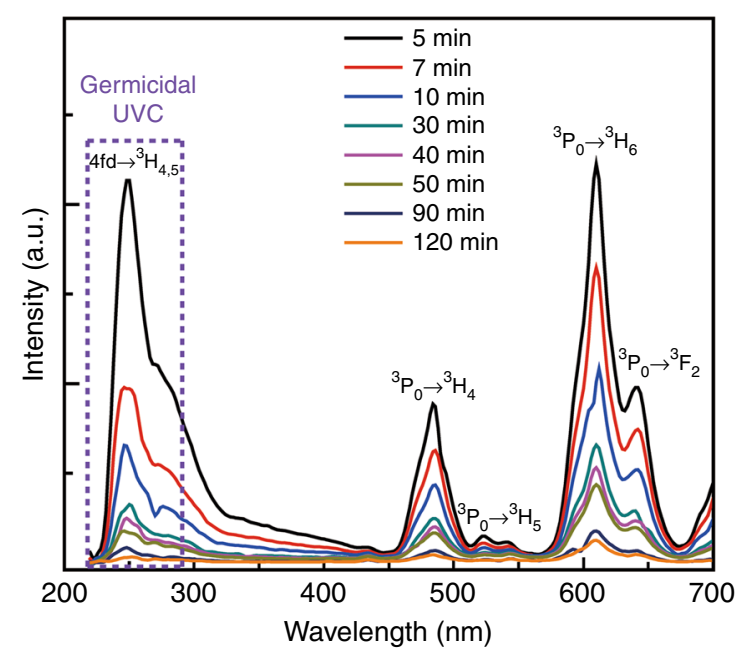

b

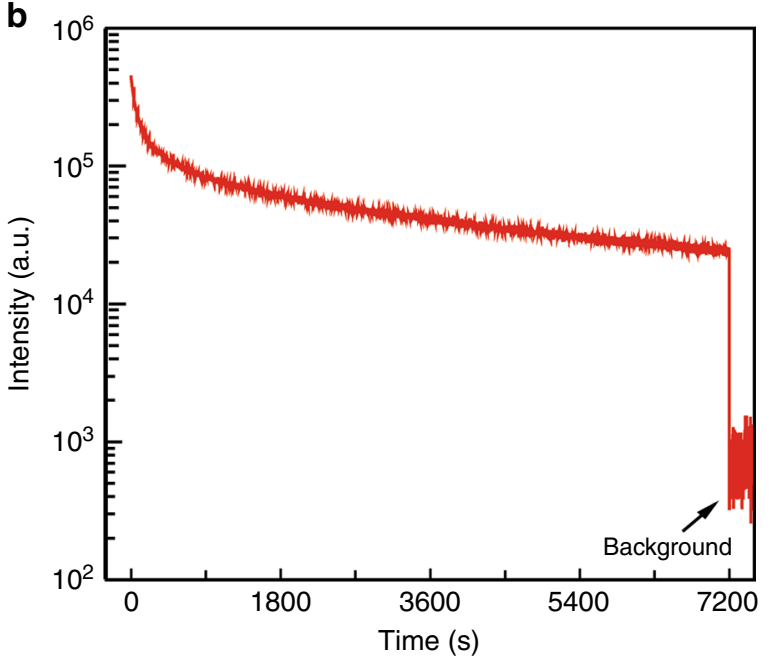

C $40 \mathrm{~s}$

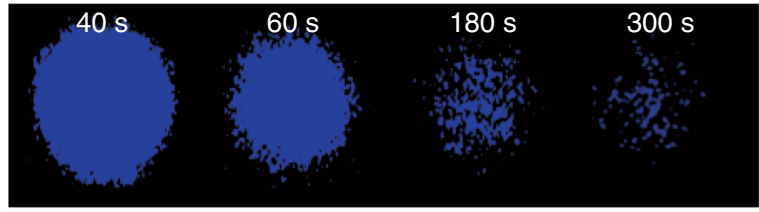

d
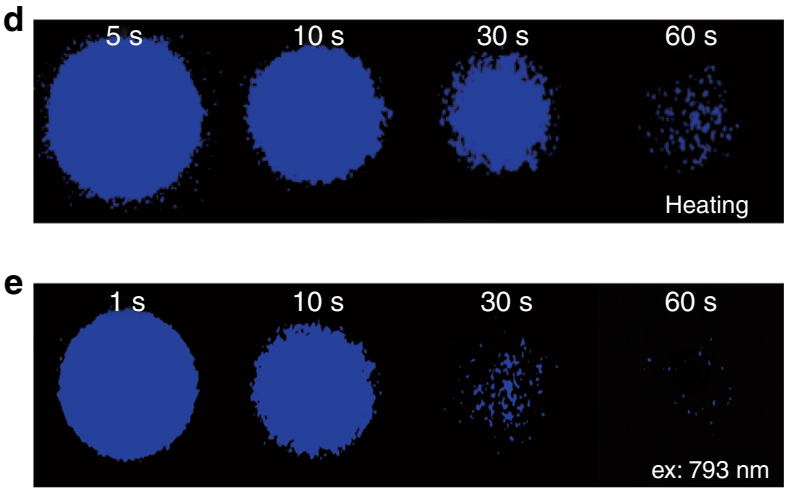

$\mathbf{f}$
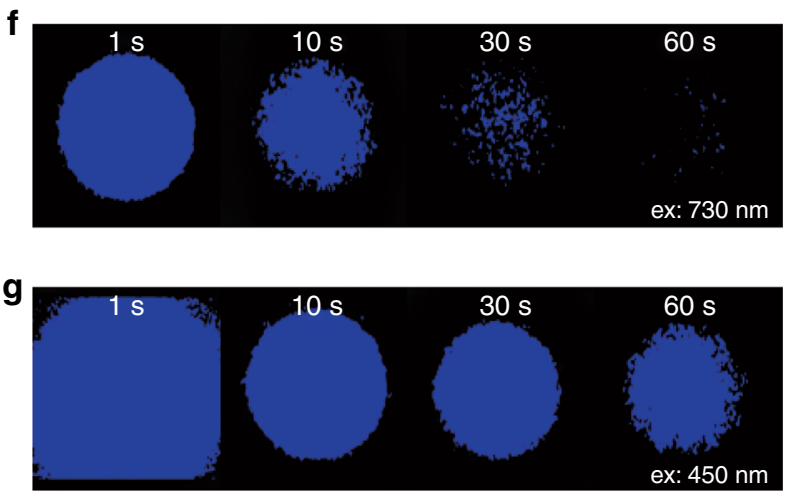

Fig. 1 UVC afterglow of $\mathrm{Pr}^{3+}$-doped fluoride elpasolite with a nominal composition of $\mathrm{Cs}_{2} \mathrm{NaY}_{0.99} \mathrm{~F}_{6}: 0.01 \mathrm{Pr}^{3+}$ and X-ray irradiation for $1000 \mathrm{~s}$. a The afterglow spectra recorded at different times after ceasing X-ray irradiation. The emission band peaking at $250 \mathrm{~nm}$ and the emission shoulder at $270 \mathrm{~nm}$ can be assigned to the transitions of $4 f 5 d \rightarrow{ }^{3} \mathrm{H}_{4}$ and $4 f 5 d \rightarrow{ }^{3} \mathrm{H}_{5}$, respectively. In addition to the UVC emissions, visible emission bands were also observed. b Afterglow decay detected at $250 \mathrm{~nm}$ as a function of time. The data were taken from 5 min after stopping the X-ray irradiation. c UVC images of phosphors taken at different afterglow times; the images after $300 \mathrm{~s}$ are shown in Supplementary Fig. 10a. d UVC images of the 24-h-decayed phosphors heated to $200^{\circ} \mathrm{C}$ on a hot plate. $\mathbf{e - g}$ UVC images of the 24-h-decayed phosphors under laser irradiation with different wavelengths of (e) $793 \mathrm{~nm},(\mathbf{f}) 730 \mathrm{~nm}$, and (g) $450 \mathrm{~nm}$. The excitation power density is $1.77 \mathrm{~W} / \mathrm{cm}^{2}$ for the 793, 730, and 450 nm excitation wavelengths 


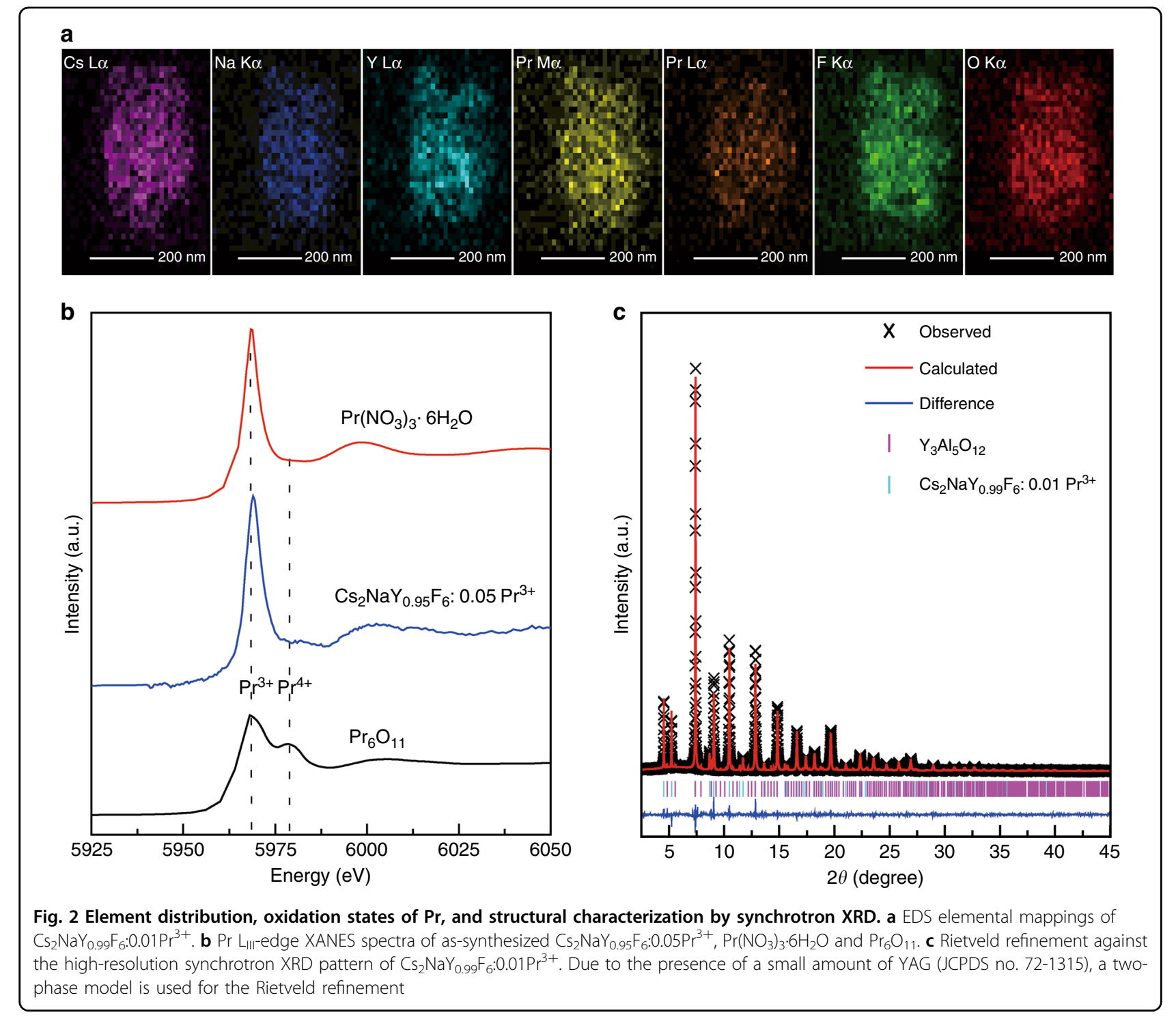

stored electrons partly in the form of UVC photons under NIR-light stimuli renders it attractive for the in vivo killing of pathogens and cancer cells.

The above results clearly show that $\operatorname{Pr}^{3+}$ ions act as emitters, but the identity of the traps remains unclear in this persistent phosphor. To understand the mechanism of persistent luminescence observed here, we next performed detailed experimental characterizations of the composition, structure, and possible defects in this system using a wide range of techniques. The composition of the yielded powders was first characterized by transmission electron microscopy and energy-dispersive X-ray spectroscopy (TEM-EDS). Interestingly, in addition to the presence of the expected constituent elements of $\mathrm{Cs}_{2} \mathrm{NaY}_{0.99} \mathrm{~F}_{6}: 0.01 \mathrm{Pr}^{3+}$, we find that the oxygen element is nearly homogeneously distributed in the particle (Fig. 2a). This elemental distribution was also verified by the scanning electron microscopy (SEM)-EDS measurements (Supplementary Fig. 12), yielding an average O/F molar ratio of $12.3 \%$. To ascertain that the oxygen distribution is not limited to the surface region, we also performed the X-ray photoelectron spectroscopy (XPS) measurements. As illustrated in Supplementary Fig. 13, the phosphor has a significant amount of oxygen, which is observed even after argon plasma etching to remove the outer surface layer. This suggests that the oxygen atoms extend throughout the bulk of the material instead of merely on the surface. We surmise that this distribution may be caused by the insufficient fluorination of oxide precursors by $\mathrm{NH}_{4} \mathrm{~F}$ during synthesis. Additionally, given that Pr ions are expected to play a role in the energy harvest and release within this UVC persistent phosphor, we next probed the oxidation state of $\operatorname{Pr}$ ions by $\operatorname{Pr} \mathrm{L}_{\mathrm{III}}$-edge X-ray absorption near-edge structure (XANES). Two typical 
compounds containing $\operatorname{Pr}, \operatorname{Pr}\left(\mathrm{NO}_{3}\right)_{3} \cdot 6 \mathrm{H}_{2} \mathrm{O}$, and $\operatorname{Pr}_{6} \mathrm{O}_{11}$, were employed as reference samples. As displayed in Fig. 2b, the XANES spectra signify the absence of $\operatorname{Pr}^{4+}$ ions in the as-synthesized phosphor, suggesting the isovalent substitution of $\operatorname{Pr}^{3+}$ for $\mathrm{Y}^{3+}$ ions.

To obtain more information concerning the structure of the phosphor, we performed a high-resolution synchrotron XRD measurement. Most of the diffraction pattern is readily indexed with an $\mathrm{Fm}-3 \mathrm{~m}$ space group, and some weak extra diffraction lines assigned to yttrium aluminum garnet (YAG) were detected. The occurrence of YAG in the product results from the reaction between the precursors and the corundum boat used for the synthesis. We note that the afterglow performance of phosphors synthesized using different corundum boats does not show much difference (Supplementary Fig. 14), suggesting good reproducibility. Rietveld refinement based on a two-phase model was performed using the general structure analysis system (GSAS) software package ${ }^{34}$. Assuming the Pr at the $\mathrm{Y}$ site and the $\mathrm{O}$ and $\mathrm{F}$ occupying the same Wyckoff site, the Rietveld refinement of the data immediately converged to $R_{\mathrm{p}}=4.38 \%$ and $R_{\mathrm{wp}}=5.91 \%$ (Fig. 2c, Table S1). The site occupancy factor for the $\mathrm{F}$ and $\mathrm{O}$ atoms was also refined and was determined to be $0.956(3)$, resulting in a chemical formula of $\mathrm{Cs}_{2} \mathrm{NaY}(\mathrm{Pr}) \mathrm{F}$ $(\mathrm{O})_{5.736} \square_{0.264}$, where $\square$ represents anion vacancies. We stress that the existence of a trace amount of $\mathrm{Gd}^{3+}$ in the phosphor, owing to the unavoidable contaminant by the precursors (the $\mathrm{Y}$ and/or Pr precursors), does not notably affect the afterglow behavior (Supplementary Fig. 15a). The occurrence of a large number of fluorine vacancies can be tentatively attributed to the replacement of $\mathrm{F}^{-}$by $\mathrm{O}^{2-}$ that forces the release of $\mathrm{F}^{-}$to satisfy the charge neutrality. We also stress that the $\operatorname{Pr}^{4+}$ ions are absent in both as-synthesized and charged products, as suggested by the XANES and electron spin resonance (ESR) spectroscopy, respectively (Fig. 2b, Supplementary Fig. 15b). Based on these information, we speculate that the chemical formula of the as-synthesized product can be written as $\mathrm{Cs}_{2} \mathrm{NaY}_{0.99} \mathrm{Pr}_{0.01} \mathrm{~F}_{5.472} \mathrm{O}_{0.264} \square_{0.264}$. We point out that this corresponds to a molar ratio of oxygen to fluorine of $13.0 \%$ when considering the existence of YAG in the product (Table S1), which is comparable to that by the EDS measurement. Collectively, the structural analysis clearly justifies the existence of anion vacancies in the product, which are thus conceived to act as electron traps.

To ascertain the possibility of anion vacancy-mediated trapping of electrons in our product, we performed density functional theory (DFT) calculations. We underscore that there are many possibilities for defects or defect complexes in this type of mixed-anion system with vacancies. To simplify the discussion, we primarily focused on the effect of fluorine vacancies on the electronic structure of $\mathrm{Cs}_{2} \mathrm{NaYF}_{6}$. We first considered three models for defective $\mathrm{Cs}_{2} \mathrm{NaYF}_{6}$ featuring a single fluorine vacancy at the apical site of the $\left[\mathrm{YF}_{6}\right]$ octahedron and two fluorine vacancies at two apical sites or at one apical and one equatorial site of the $\left[\mathrm{YF}_{6}\right]$ octahedron. We note that the calculated band gap for the pristine $\mathrm{Cs}_{2} \mathrm{NaYF}_{6}$ is 9.67 $\mathrm{eV}$, which is comparable to the experimental value ${ }^{33}$. The calculated density of states (DOS) for both pristine and defective $\mathrm{Cs}_{2} \mathrm{NaYF}_{6}$ is shown in Fig. 3a-d. Interestingly, we find that the creation of one fluorine vacancy at the apical site of the octahedron introduces four in-gap states, at approximately $0.18,0.60,1.45$, and $2.86 \mathrm{eV}$ below the conduction band minimum (CBM) (Fig. 3b). Similarly, creating two fluorine vacancies at two apical sites of one $\left[\mathrm{YF}_{6}\right]$ octahedron gives rise to three in-gap states, at approximately $0.24,1.26$, and $2.85 \mathrm{eV}$ below the CBM (Fig. 3c). By contrast, the formation of two fluorine vacancies at one apical and one equatorial site of the octahedron leads to more complex in-gap states, with the deepest defect level being $3.11 \mathrm{eV}$ below the CBM (Fig. 3d). We also calculated the DOS of the defective $\mathrm{Cs}_{2} \mathrm{NaYF}_{6}$ with two fluorine vacancies at two apical sites or at one apical and one equatorial site of the $\left[\mathrm{NaF}_{6}\right]$ octahedron, and we found similar in-gap states (Supplementary Fig. 16). All these results unambiguously indicate that the introduction of fluorine vacancies in $\mathrm{Cs}_{2} \mathrm{NaYF}_{6}$ results in the appearance of a series of in-gap defect levels with different depths with respect to the CBM. We stress that the deeper traps with $>1.3 \mathrm{eV}$ below the CBM predicted here can be supported well by the photostimulated luminescence as shown in Fig. 1e-g.

The existence of fluorine vacancy-related defect levels in our product was also experimentally confirmed by the thermoluminescence measurements. Figure 4a displays the thermoluminescence curve of the persistent phosphor at $48 \mathrm{~h}$ after stopping the X-ray irradiation. The trap depths $(E)$ relative to the CBM can be calculated by the expression $E=0.002 T_{\mathrm{m}}$, in which $T_{\mathrm{m}}$ is the temperature for which the thermoluminescence peak is the maximum (in kelvin, K) $)^{2}$. Three shallow traps, with respect to those $>1.3 \mathrm{eV}$ below the CBM, were observed to have the activation energy values of $0.69,0.83$, and $1.02 \mathrm{eV}$ at 70 , 141 , and $238^{\circ} \mathrm{C}$, respectively. We note that the determined shallow defects have good consistency with the results calculated by DFT (Fig. 3b-d), although other structural defects, beyond the cases in our DFT calculations (e.g., the formation of $\left[\mathrm{YF}_{3}\right]$ or $\left[\mathrm{NaF}_{3}\right]$ due to the loss of three fluorine in one octahedron or the formation of defect complex consisting of $\left[\mathrm{YF}_{5}\right],\left[\mathrm{NaF}_{5}\right]$ or others), probably also contribute to the formation of these shallow traps.

On the basis of all these results, we posit that the defects associated with fluorine vacancies could act as electron trapping centers with diverse depths with respect to the CBM, making fluoride elpasolite an excellent X-ray- 

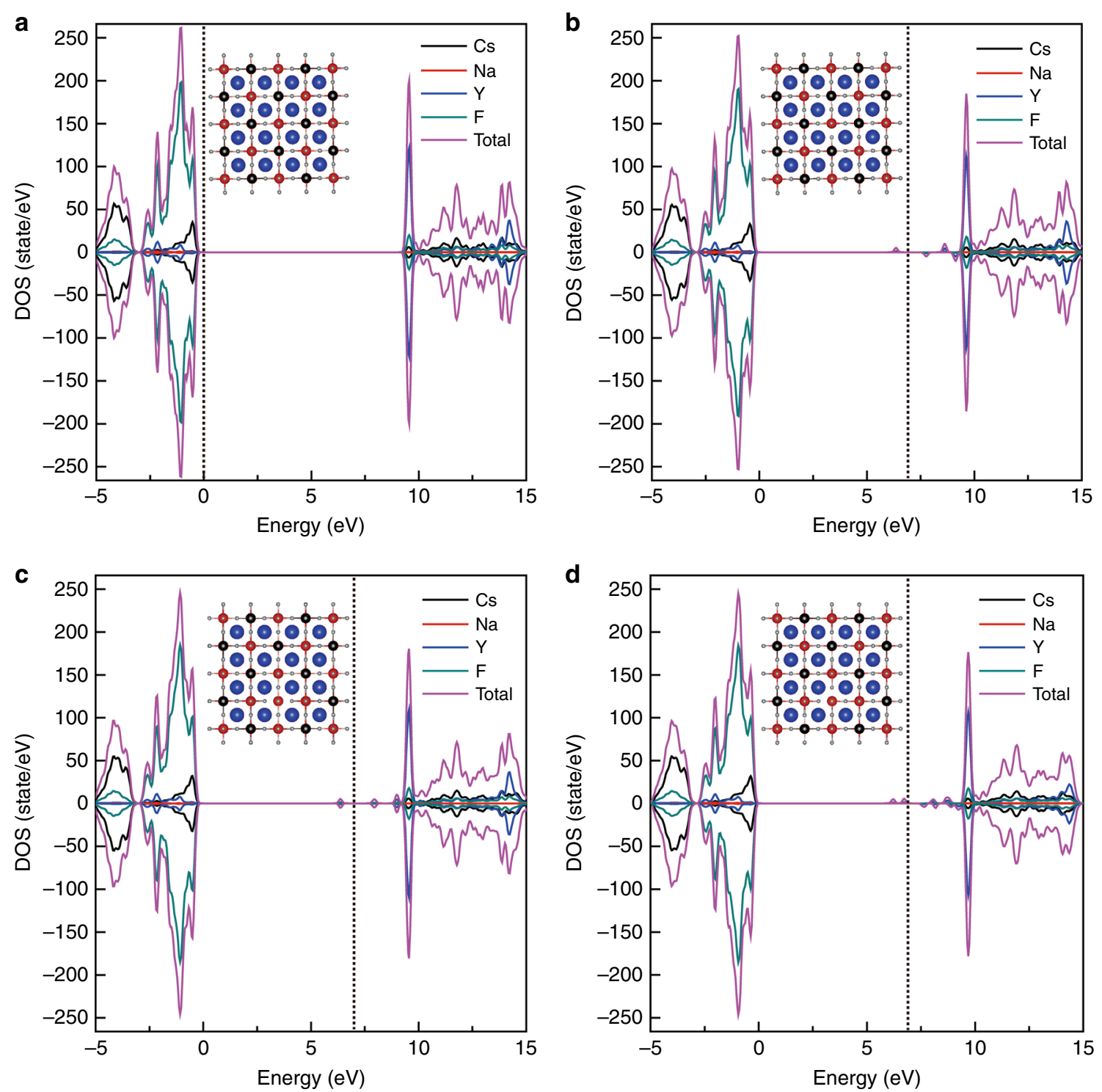

Fig. 3 DOS of pristine and defective $\mathrm{Cs}_{2} \mathrm{NaYF}_{6}$. a DOS of pristine $\mathrm{CS}_{2} \mathrm{NaYF}_{6}$. $\mathbf{b}$ DOS of $\mathrm{CS}_{2} \mathrm{NaYF}_{6}$ with one fluorine vacancy at the apical site of the $\left[\mathrm{YF}_{6}\right]$ octahedron. $\mathbf{c} \mathrm{DOS}$ of $\mathrm{Cs}_{2} \mathrm{NaYF}_{6}$ with two fluorine vacancies at two apical sites of one $\left[\mathrm{YF}_{6}\right]$ octahedron. $\mathbf{d}$ DOS of $\mathrm{Cs}_{2} \mathrm{NaYF}_{6}$ with two fluorine vacancies at one apical and one equatorial site of the $\left[\mathrm{YF}_{6}\right]$ octahedron. The insets in (a-d) show the structures for the calculations, where blue, black, red, and pale spheres represent $\mathrm{Cs}, \mathrm{Na}, \mathrm{Y}$, and $\mathrm{F}$ ions, respectively. The vertical dotted lines in (a-d) represent the Fermi level. The zero-energy point was set to the Fermi level of pristine $\mathrm{Cs}_{2} \mathrm{NaYF}_{6}$. The Fermi levels of the defect-bearing systems were corrected by aligning the average electrostatic potential $\left(V_{\text {av }}\right)$ of $F$ atoms located far from the defects to the $V_{a v}$ of the same elements in the pristine $\mathrm{Cs}_{2} \mathrm{NaYF}_{6}$

excitable UVC long-lasting phosphor. The use of X-ray as the excitation source signifies that the dominant mechanism for the persistent luminescence observed is by the direct recombination of released electrons with $\mathrm{Pr}^{3+}$ ions. Nevertheless, the fact that the visible persistent luminescence decays more slowly than the UVC, both of which are due to the electronic transitions of $\mathrm{Pr}^{3+}$, indicates that other processes should also involve the electron detrapping. In view of the existence of the YAG phase in the product which may influence the afterglow, we further synthesized the sample using a platinum crucible; the XRD result suggests the absence of any impurity in the product (Supplementary Fig. 17a). Interestingly, we note that this phase-pure sample shows similar visible PL, but different photoluminescence excitation spectra with respect to the YAG-containing phosphor (Supplementary Fig. 17b,c and Supplementary Fig. 4b). It is well known that the absorption wavelength of ${ }^{3} \mathrm{H}_{4} \rightarrow 4 f 5 d$ of $^{\mathrm{Pr}^{3+}}$ in YAG is longer than that in the elpasolite ${ }^{33,35}$, suggesting that the UVC emissions observed in the YAG-containing 
phosphor originate from the elpasolite phase and that the observed photoluminescence excitation band in Supplementary Fig. 4 mainly originates from the YAG phase. Additionally, we find that, at $5 \mathrm{~min}$ after stopping the $\mathrm{X}$ ray irradiation, the relative intensity of the UVC to the visible emissions for the YAG-containing sample is smaller than that for the phase-pure product (Supplementary Fig. 17d), which signifies that the YAG phase, along with the elpasolite phase, contributes to the visible afterglow. We underscore that, similar to the YAGcontaining sample, the UVC afterglow decays faster than the visible cousin in the phase-pure sample (Supplementary Fig. 17e). The decay curve corresponding to the visible emission was further plotted as a function of reciprocal persistent luminescence intensity $\left(I^{-1}\right)$ versus time $(t)$ (Supplementary Fig. 17f). The $I^{-1} \sim t$ at the 50-120 min period for the red persistent luminescence can be fitted linearly, suggesting that a tunneling-related process occurs ${ }^{11}$. Based on all these observations, we propose a plausible mechanism for the UVC and visible persistent luminescence, as schematized in Fig. 4b. Upon $\mathrm{X}$-ray irradiation, the absorption of an X-ray photon yields an energetic, ionized free electron. This hot electron collides with atoms in the material, and it triggers the cascading production of additional ionized electrons ${ }^{36}$. Lower-energy collisions may cause the excitation of valence band electrons into the conduction band, leading to the creation of many electron-hole pairs (process 1 in Fig. 4b). The excited electrons and created holes are subsequently captured by electron traps and $\mathrm{Pr}^{3+}$ ions, respectively, based on processes 2 and 3. After long-term
$\mathrm{X}$-ray irradiation, the traps are filled. After X-ray irradiation ceases, at the beginning, the electrons are primarily released from shallow traps, followed by transfer to the $\mathrm{Pr}^{3+}$ ions through the conduction band (process 4). The $\operatorname{Pr}^{3+}$ ion with an electron and a hole can be viewed as an excited $\mathrm{Pr}^{3+}$, which releases the energy either through the $4 f 5 d-4 f^{2}$ transition or through the $4 f^{2}-4 f^{2}$ transition (process 5) that cause the UVC and visible afterglow, respectively. After the depletion of electrons captured in shallow traps, those in the deep traps can migrate directly to nearby $\operatorname{Pr}^{3+}$ ions by tunneling and then they are captured by the $4 f^{2}$ energy levels of $\operatorname{Pr}^{3+}$, resulting in the visible emissions in the absence of the UVC emission (process 6). We point out that overexposing the phosphor under X-rays leads to weaker UVC emission (Supplementary Fig. 7). This may be caused by some X-rayinduced defects ${ }^{37}$, which remains an open question for further study. We stress that after releasing most of the stored electrons, the phosphor can be recharged, showing a nearly identical afterglow behavior (Supplementary Fig. 18).

\section{Discussion}

Here, we present the discovery of UVC persistent luminescence in a defective, $\operatorname{Pr}^{3+}$-doped fluoride elpasolite, and we demonstrate that the development of UVC persistent phosphors is not an insurmountable goal. Specifically, we have found that incorporating oxygen into the lattice results in the formation of a large number of anion vacancies that can serve as electron traps. The extension of the spectral range of persistent luminescence
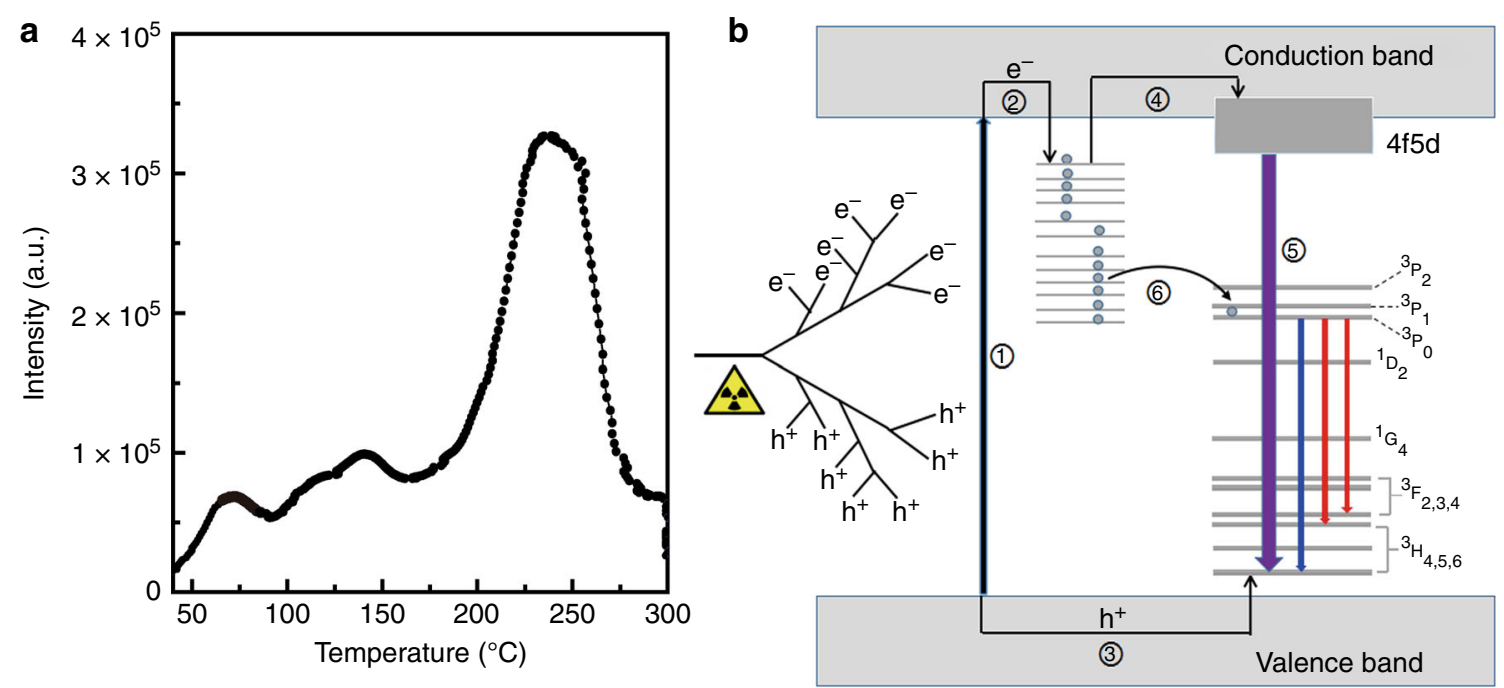

Fig. 4 Thermoluminescence spectrum and schematic illustration of the proposed afterglow mechanism. a The thermoluminescence spectrum of phosphors with a nominal composition of $\mathrm{Cs}_{2} \mathrm{NaY}_{0.99} \mathrm{~F}_{6}: 0.01 \mathrm{Pr}^{3+}$. The sample was irradiated for $1000 \mathrm{~s}$ at room temperature, and then was left for $48 \mathrm{~h}$ before the thermoluminescence measurement. b Proposed afterglow mechanism. The purple, blue, and red lines represent the optical transitions corresponding to the UVC, blue, and red emissions, respectively. Note that the emission corresponding to the transition of $4 f 5 d \rightarrow{ }^{3} \mathrm{H}_{5}$ is partially in the UVC spectral range 

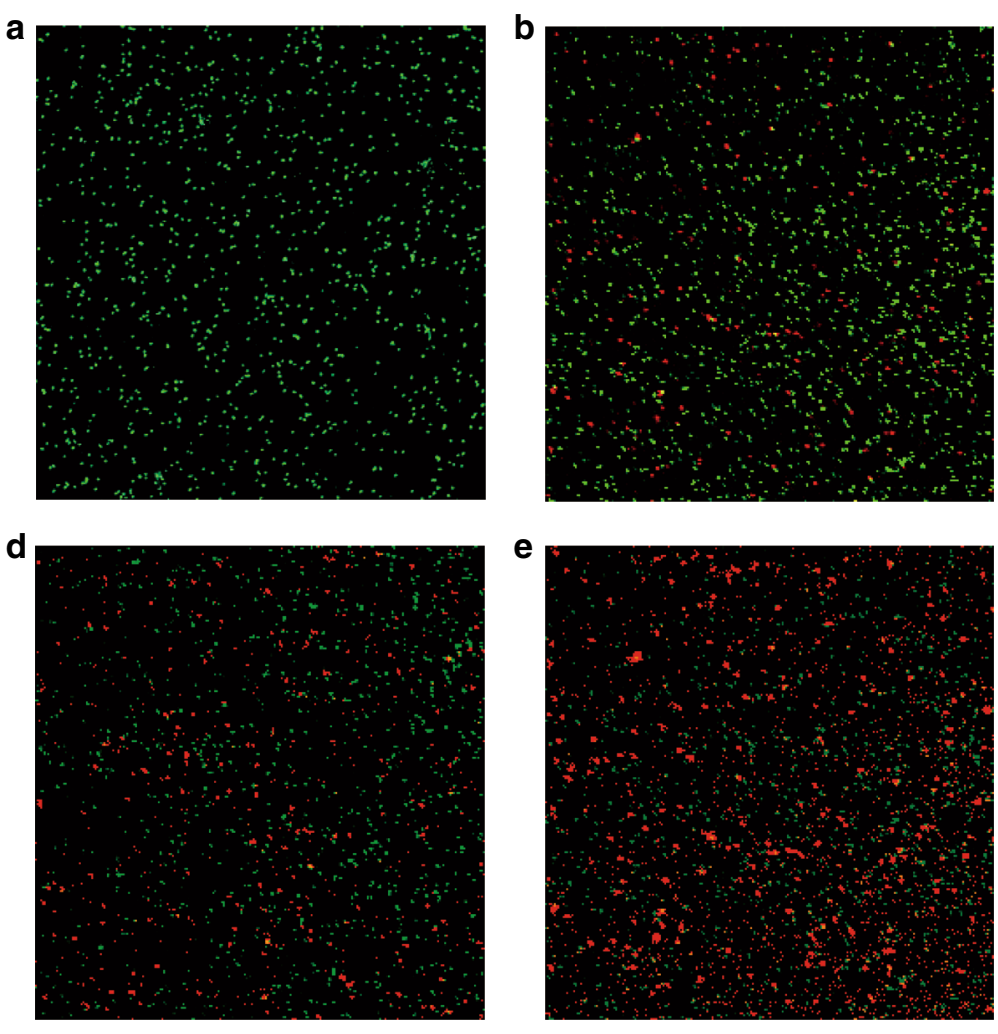
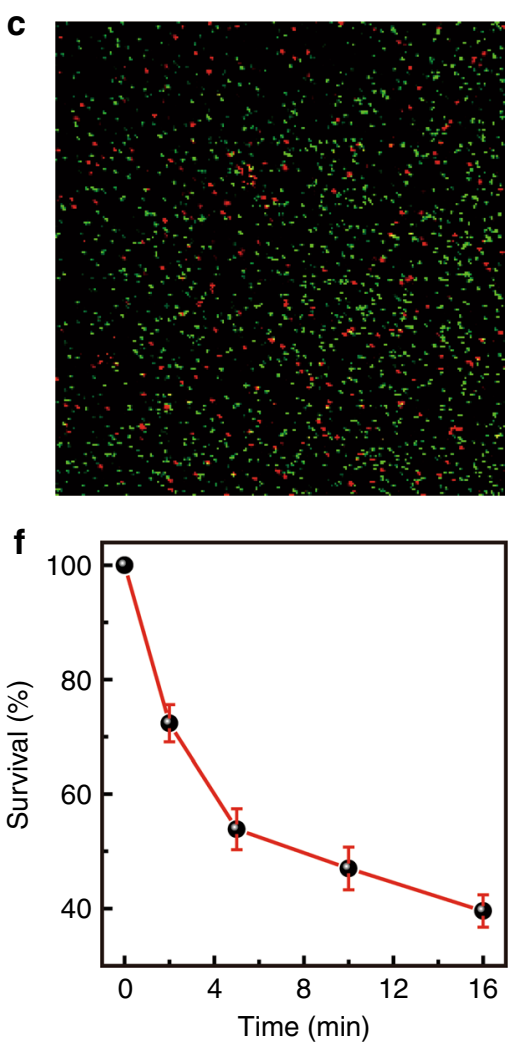

Fig. 5 Inactivation of $P$. aeruginosa PAO1 using UVC-afterglow phosphor sheets. a Confocal micrograph of the control sample exposed at ambient conditions. b-e Confocal micrographs of $P$. aeruginosa PAO1 undergoing different irradiation of UVC afterglow. Four phosphor sheets that were irradiated by X-ray for (b) $2 \mathrm{~min}$, (c) $5 \mathrm{~min}$, (d) $10 \mathrm{~min}$, and (e) $16 \mathrm{~min}$ were used to inactivate $P$. aeruginosa PAO1. The live and dead cells show green and red colors, respectively. $\mathbf{f}$ The dependence of $P$. aeruginosa PAO1 survival ratios on the X-ray irradiation time of the given phosphor sheets. $100 \%$ viability can be maintained when keeping P. aeruginosa PAO1 under ambient conditions. The error bars represent the standard error and are obtained based on three independent experiments

from visible and NIR to UVC opens up a diversity of potential applications.

As is well known, Pseudomonas $(P$.$) aeruginosa PAO1 is$ a common Gram-negative and monoflagellated bacterium that can survive in a diversity of environment conditions ${ }^{38}$. The organism can cause disease not only in animals and plants but also in humans. As a proof of concept, we show that the UVC persistent phosphor developed here can be used for killing $P$. aeruginosa PAO1. As shown in Fig. 5a, 100\% viability can be maintained when keeping $P$. aeruginosa PAO1 under ambient conditions (i.e., room light, normal atmosphere) for $30 \mathrm{~min}$. We used a total of four sheets of UVC persistent phosphors that were irradiated by X-rays for $2,5,10$, and $16 \mathrm{~min}$, respectively. The intensity of the persistent luminescence increases with the increasing irradiation time. We note that before irradiation, each sheet was fixed on a $\mathrm{u}$-shaped bracket. At $2 \mathrm{~s}$ after the cease of irradiation, the longpersistent luminescent sheet was kept close to the culture dish. Interestingly, we observe that the survival of $P$. aeruginosa $\mathrm{PAO} 1$ is associated with the $\mathrm{X}$-ray irradiation time of the sheet, and around $39.6 \%$ viability can be maintained for $P$. aeruginosa PAO1 in the dish with the sheet after $16 \mathrm{~min}$ of irradiation (Fig. 5b-f). This observation provides direct evidence that the persistent luminescence from our UVC phosphor can be used for sterilization. Thanks to the outstanding penetrating ability of X-rays, the charging of our phosphors are barely influenced by biological tissues (Supplementary Fig. 19), thus highlighting its enormous potential for in vivo applications.

\section{Conclusions}

In summary, we have developed a new class of phosphor that exhibits strong and long-lasting UVC afterglow. A combination of experimental and theoretical results leads us to propose that the structural defects in the elpasolite that are associated with oxygen introduction-induced anion vacancies serve as electron traps, which render it capable of capturing and storing a large number of electrons as triggered by X-ray irradiation. Interestingly, the afterglow intensity of this phosphor is sufficiently strong 
for sterilization. We believe that the concept shown here may be applicable to other $\operatorname{Pr}^{3+}$-doped wide-bandgap compounds, suggesting a series of UVC persistent phosphors with excellent performance. Our finding of this UVC afterglow opens up a new frontier in persistent phosphors, and offers an opportunity for novel applications, such as sterilization, disinfection, drug release, the in vivo killing of cancer cells, anti-counterfeiting, and beyond.

\section{Materials and methods}

\section{Synthesis of UVC persistent phosphors}

Pr-doped polycrystalline fluoride elpasolite phosphors, with nominal compositions of $\mathrm{Cs}_{2} \mathrm{NaY}_{(1-x)} \mathrm{F}_{6}: x \mathrm{Pr}^{3+}$, were prepared by a solid-state reaction method. $\mathrm{Cs}_{2} \mathrm{CO}_{3}$ (1.6290 g, 99.99\%, Aladdin, Shanghai, China), $\mathrm{NaHCO}_{3}$ (0.4200 g, 99.99\%, Aladdin, Shanghai, China), $\mathrm{Y}_{2} \mathrm{O}_{3}$ (0.5588 g, 99.99\%, Aladdin, Shanghai, China), $\mathrm{NH}_{4} \mathrm{~F}$ (2.2222 g, 99.99\%, Aladdin, Shanghai, China), and $\operatorname{Pr}_{6} \mathrm{O}_{11}$ $(0.0085 \mathrm{~g}, 99.996 \%$, Alfa, United States $)$ powders were mixed together with $3 \mathrm{~mL}$ of acetone and then ground thoroughly. The obtained powders were thermally treated at $150^{\circ} \mathrm{C}$ in air for $7 \mathrm{~h}$, followed by regrinding to obtain a fine powder. The mixture was first sintered at $450{ }^{\circ} \mathrm{C}$ for $30 \mathrm{~min}$ in air atmosphere. The obtained powders were then reground, followed by sintering at $700{ }^{\circ} \mathrm{C}$ for $10 \mathrm{~h}$ under a nitrogen atmosphere. The white powders were collected and stored for further characterizations. Corundum boats with a purity of $99 \%$ and a platinum crucible were used as vessels for the above synthesis.

\section{Charging of persistent phosphors}

The X-ray irradiation of the product was performed using a calibrated RS-2000 biological irradiator equipped with a tungsten target $(160 \mathrm{kV}, 25 \mathrm{~mA})$, and the $\mathrm{X}$-ray dose was tuned by changing the irradiation time. The wavelength of X-ray from the irradiator is $0.2106 \AA$.

\section{Structural and morphological characterizations}

XRD patterns were taken using an X'Pert-Pro MPD diffractometer (Netherlands PANalytical) with a $\mathrm{Cu} \mathrm{K \alpha}$ X-ray source $(\lambda=1.540598 \AA$ ). TEM images and TEMEDS mapping were taken with an FEI Tecnai G2 F20 STWIN TMP microscope $(200 \mathrm{kV})$. SEM image and SEM-EDS mapping were taken with a Zeiss scanning electron microscope (Zeiss Supra55). We note that three different regions with a size of $\sim 5 \mu \mathrm{m} \times 5 \mu \mathrm{m}$ for the samples were used for the EDS measurement; the O/F ratio was obtained by averaging these data, and determined to be $(12.3 \pm 2.0) \%$. XPS was performed on a Rigaku XPS-7000 spectrometer. The carbon peak at $284.6 \mathrm{eV}$ was used as a reference to correct the charging effect.

\section{Luminescence, afterglow, and thermoluminescence characterizations}

Luminescence spectra were recorded by a spectrofluorometer (FLS980, Edinburgh Instruments Ltd.) equipped with a photomultiplier (R928P with an applied voltage of $950 \mathrm{~V}$, Hamamatsu). The persistent luminescence spectra were taken at different time intervals after ceasing the X-ray irradiation. All data regarding the afterglow intensity versus time were recorded from $5 \mathrm{~min}$ after stopping the X-ray irradiation. Note that the slit widths of the detection monochromator used for the luminescence and afterglow measurements are $3 \mathrm{~nm}$ and $10 \mathrm{~nm}$, respectively, which results in relatively broad afterglow emission bands with respect to photoluminescence bands (Figs. 1a and S4a). Thermoluminescence measurements were performed with a thermoluminescent dosimeter (FJ-427A1), with a heating rate of $1{ }^{\circ} \mathrm{C} / \mathrm{s}$ from room temperature to $300^{\circ} \mathrm{C}$. The sample was irradiated for $1000 \mathrm{~s}$ at room temperature, and then it was left for $48 \mathrm{~h}$ before the thermoluminescence measurement.

\section{Real-time afterglow measurements by a UVC imager}

A homemade visible-blind UVC imager was used for this measurement. We note that the sensitive range of this imager is $240-280 \mathrm{~nm}$, which was achieved by adding a bandpass filter. The UVC signals from the samples, which were recorded as the number of photons, were recorded by the UVC imager. To avoid the saturation of photon counts, a relatively low applied voltage was used for recording the initial UVC images. The afterglow decay curves shown in Figs. S10b, c and S11 were drawn based on these measurements. The photostimulated luminescence was monitored by this UVC imager under the excitation of laser diodes with emissions peaking at 450, 730 , and $793 \mathrm{~nm}$. To monitor the UVC signal under heating, the sample was heated by a hot plate set to $200{ }^{\circ} \mathrm{C}$ and the UVC signal was measured by the UVC imager; note that the first image was taken at $5 \mathrm{~s}$ after putting the sample on the hot plate. The distance between the imager and the sample was $70 \mathrm{~cm}$. Both photostimulated and thermostimulated UVC images were taken at $24 \mathrm{~h}$ after ceasing X-ray irradiation (the irradiation time: $1000 \mathrm{~s}$ ). We emphasize that the sensitivity of the UVC imager used is poorer than that of photomultiplier used for afterglow measurements as shown in Fig. 1c.

\section{Estimation of the power density of the UVC afterglow}

We roughly evaluate the power density of the UVC afterglow using a Thorlabs PM200 power meter equipped a power sensor (S120VC, Thorlabs). The detailed measurement method is shown in Supplementary Fig. 20. After considering all possible factors that impact the measurement, the initial afterglow power density 
at the sample position was roughly estimated to be ca. $14.9 \mathrm{~mW} / \mathrm{m}^{2}$.

\section{Synchrotron X-ray measurement}

We took the synchrotron XRD measurement using the BL02B2 beam line of SPring-8 to obtain high-quality diffraction patterns at $296 \mathrm{~K}$. The sample was sealed into Hilgenberg glass capillaries with an inner diameter of $0.1 \mathrm{~mm}$, and during the measurement, the capillary was continuously rotating. The $\mathrm{X}$-ray wavelength used is $0.413745 \AA$. Rietveld refinement was preformed against XRD data utilizing the GSAS program ${ }^{34}$. The room temperature $\operatorname{Pr} \mathrm{L}_{\mathrm{III}}$-edge XANES was taken on the 1W1B beam line of the Beijing Synchrotron Radiation Facility with a stored electron energy of $2.5 \mathrm{GeV}$ and average ring currents of $200 \mathrm{~mA}$. A fixed-exit Si (111) double crystal monochromator was used. $\operatorname{Pr}_{6} \mathrm{O}_{11}$ and $\operatorname{Pr}\left(\mathrm{NO}_{3}\right)_{3} \cdot 6 \mathrm{H}_{2} \mathrm{O}$ powders were used as reference samples. Data were collected in the fluorescence mode for the studied sample and in the transmission mode for the reference samples. The XAFS data were analyzed using the IFEFFIT software package $^{39}$.

\section{Bactericidal experiment}

The $P$. aeruginosa PAO1 in the culture dishes with the beef extract peptone medium was grown in an incubator at $35^{\circ} \mathrm{C}$. Three days later, the PAO1 population in the culture dishes was $\sim 10^{6}$ colony-forming units $(\mathrm{cfu}) / \mathrm{mL}$. The culture dishes were removed from the incubator and used for the following inactivation experiment. To perform the sterilization experiment, the samples were tableted by a homemade tablet machine. The $\mathrm{Cs}_{2} \mathrm{NaY}_{0.99} \mathrm{~F}_{6}: 0.01 \mathrm{Pr}^{3+}$ powders with a mass of $7.4 \mathrm{~g}$ were compressed into a sheet sample of $3 \mathrm{~cm}$ in diameter and $4 \mathrm{~mm}$ in thickness. Each sheet was fixed on a u-shaped bracket, followed by X-ray irradiation for 2, 5, 10, and $16 \mathrm{~min}$. At $2 \mathrm{~s}$ after the end of irradiation, the longpersistent luminescent sheet was held close to the culture dish. The distance between the sheet surface and P. aeruginosa PAO1 is $\sim 2 \mathrm{~mm}$. After $30 \mathrm{~min}$, the afterglow sheet was removed, and then the PAO1 was diluted with deionized water and centrifuged at $3000 \mathrm{rpm}$ for $20 \mathrm{~min}$. The PAO1 was then dispersed in $20 \% \mathrm{NaCl}$ solution and used for the following test. To evaluate the cell membrane integrity, a BacLight live/dead bacterial viability kit (L-7012, Molecular Probes) was used, which allows us to differentiate cells with intact (live) membranes from those with damaged (dead) membranes. The stain was then prepared by diluting $3 \mu \mathrm{L}$ of each component into $1 \mathrm{~mL}$ of distilled water, and then it was kept in the dark for $15 \mathrm{~min}$. We note that at least 2000 cells were scored per sample for the analysis. The $P$. aeruginosa PAO1 suspension was imaged using a confocal laser scanning microscope. A water immersion objective lens was used. The
$P$. aeruginosa $\mathrm{PAO} 1$ suspension images corresponding to the afterglow sheets with X-ray irradiation for 2, 5, 10, and 16 min were compared to confirm the inactivation effect.

\section{First-principles calculations}

DFT calculations are performed using the Vienna $\mathrm{Ab}$ initio simulation package $(\mathrm{VASP})^{40}$. We used the Perdew-Berke-Ernzerhof of generalized gradient approximate (GGA) functional for the description of the exchange and correlation energy of the electrons. Because the GGA usually underestimates the band gap of materials, an orbital-dependent potential was used, including an additional Coulomb interaction (Hubbard $U)^{41-44}$. The underestimation of the intraband Coulomb interactions was corrected by the Hubbard $U$ parameter and the values of $U_{\mathrm{Cs}}(d)=8 \mathrm{eV}, U_{\mathrm{Na}}(p)=2 \mathrm{eV}, U_{\mathrm{Y}}(d)=4 \mathrm{eV}$, and $U_{\mathrm{F}}(p)=10 \mathrm{eV}$ were used. The ionic potential was described by the projector-augmented wave (PAW) pseudopotential. For $k$-point integration within the first Brillouin zone, a $2 \times 2 \times 3$ Monkhorst-Pack grid for a $2 \times 2 \times$ 1 super cell was selected. A plane-wave cutoff energy of $450 \mathrm{eV}$ was applied to the calculations. The convergence criteria for the maximum force and the total energy were set to $0.01 \mathrm{eV}^{-1}$ and $1.0 \times 10^{-4} \mathrm{eV} /$ atom, respectively. Based on the static states mentioned above, the DOS of pristine and defective $\mathrm{Cs}_{2} \mathrm{NaYF}_{6}$ was calculated.

\section{Acknowledgements}

This work was supported by the National Natural Science Foundation of China (Grant nos. 11574225, 11874275, 51672106, and 11474083) and a project funded by the Priority Academic Program Development of Jiangsu Higher Education Institutions (PAPD). We thank the staff at the 1W1B beamline at the Beijing Synchrotron Radiation Facility for the XAFS measurements. The SPring8 experiment was carried out with the approval of the Japan Synchrotron Radiation Research Institute (JASRl; Proposal no. 2017B1309).

\section{Author details}

${ }^{1}$ College of Physics Science and Technology, Hebei University, 071002 Baoding, China. ${ }^{2}$ College of Chemistry, Chemical Engineering and Materials Science, Soochow University, 215123 Jiangsu, China. ${ }^{3}$ Department of Physics, Beihang University, 100191 Beijing, China. ${ }^{4}$ Department of Physical Science, Hiroshima University, Higashihiroshima, Hiroshima 739-8526, Japan. ${ }^{5}$ Shanghai Institute of Optics and Fine Mechanics, Chinese Academy of Sciences, 201800 Shanghai, China. ${ }^{6}$ Beijing Synchrotron Radiation Facility, Institute of High Energy Physics, Chinese Academy of Sciences, 100049 Beijing, China

\section{Author contributions}

H.-T.S. conceived and supervised the research. H.-T.S., Y.-M.Y., and Z.-Y.L. designed the experiments. Z.-Y.L. performed most of the experiments and data analysis. Q.Z., H.T.S., Y.K., and C.M. took the synchrotron XRD. Y.-M.Y. and X.W. carried out the bactericidal experiment. Z.-Y.L. evaluated the power density of the UVC afterglow. Z.-Y.L. and L.-R.Z. took the XANES measurement. J.-Y.Z., Y.L., and S.-Q.G. performed the DFT calculations. Z.-J.Y., H.L., and J.-P.M. participated in the material synthesis and afterglow measurements. Z.-Y.L., L.-L.H., and L.-Y.Z. synthesized the sample using a platinum crucible. H.T.S. wrote the manuscript with input from other authors. All the authors discussed the results and commented on the manuscript.

Conflict of interest

The authors declare that they have no conflict of interest. 
Supplementary information is available for this paper at https://doi.org/ 10.1038/s41377-018-0089-7.

Received: 10 May 2018 Revised: 26 October 2018 Accepted: 26 October 2018

Published online: 14 November 2018

\section{References}

1. Hölsä, J. Persistent luminescence beats the afterglow: 400 years of persistent luminescence. Electrochem. Soc. Interface 18, 42-45 (2009).

2. Pan, Z. W., Lu, Y. Y. \& Liu, F. Sunlight-activated long-persistent luminescence in the near-infrared from $\mathrm{Cr}^{3+}$-doped zinc gallogermanates. Nat. Mater. 11, 58-63 (2001)

3. Jia, W. Y., Yuan, H. B., Lu, L. Z., Liu, H. M. \& Yen, W. M. Phosphorescent dynamics in $\mathrm{SrAl}_{2} \mathrm{O}_{4}: \mathrm{Eu}^{2+}, \mathrm{Dy}^{3+}$ single crystal fibers. J. Lumin. 76-77, 424-428 (1998).

4. Aitasalo, T. et al. Persistent luminescence phenomena in materials doped with rare earth ions. J. Solid State Chem. 171, 114-122 (2003).

5. Wang, X. J., Jia, D. D. \& Yen, W. M. Mn ${ }^{2+}$ activated green, yellow, and red long persistent phosphors. J. Lumin. 102-103, 34-37 (2003).

6. Clabau, F. et al. Mechanism of phosphorescence appropriate for the longlasting phosphors $\mathrm{Eu}^{2+}$-doped $\mathrm{SrAl}_{2} \mathrm{O}_{4}$ with codopants $\mathrm{Dy}^{3+}$ and $\mathrm{B}^{3+}$. Chem. Mater. 17, 3904-3912 (2005).

7. Aitasalo, T., Hölsä, J., Jungner, H., Lastusaari, M. \& Niittykoski, J. Thermoluminescence study of persistent luminescence materials: $\mathrm{Eu}^{2+}$ - and $\mathrm{R}^{3+}$-doped calcium aluminates, $\mathrm{CaAl}_{2} \mathrm{O}_{4}: \mathrm{Eu}^{2+}, \mathrm{R}^{3+}$. J. Phys. Chem. B 110, 4589-4598 (2006)

8. Van den Eeckhout, K., Smet, P. F. \& Poelman, D. Persistent luminescence in $\mathrm{Eu}^{2+}$-doped compounds: a review. Materials 3, 2536-2566 (2010).

9. Rodríguez Burbano, D. C., Sharma, S. K., Dorenbos, P., Viana, B. \& Capobianco, J. A. Persistent and photostimulated red emission in $\mathrm{CaS}_{\mathrm{Eu}}{ }^{2+}, \mathrm{Dy}^{3+}$ nanophosphors. Adv. Opt. Mater. 3, 551-557 (2015).

10. Norrbo, I. et al. Lanthanide and heavy metal free long white persistent luminescence from Ti doped Li-hackmanite: a versatile, low-cost material. Adv. Funct. Mater. 27, 1606547 (2017)

11. Trojan-Piegza, J., Niittykoski, J., Hölsä, J. \& Zych, E. Thermoluminescence and kinetics of persistent luminescence of vacuum-sintered $\mathrm{Tb}^{3+}$-Doped and $\mathrm{Tb}^{3+}, \mathrm{Ca}^{2+}$-codoped $\mathrm{Lu}_{2} \mathrm{O}_{3}$ materials. Chem. Mater. 20, 2252-2261 (2008).

12. Zhuang, Y. X., Wang, L., Lv, Y., Zhou, T. L. \& Xie, R. J. Optical data storage and multicolor emission readout on flexible films using deep-trap persistent luminescence materials. Adv. Funct. Mater. 28, 1705769 (2018).

13. Matsuzawa, T., Aoki, Y., Takeuchi, N. \& Murayama, Y. A new long phosphorescent phosphor with high brightness, $\mathrm{SrAl}_{2} \mathrm{O}_{4}: \mathrm{Eu}^{2+}, \mathrm{Dy}^{3+}$. J. Electrochem. Soc. 143, 2670-2673 (1996).

14. Yamamoto, H. \& Matsuzawa, T. Mechanism of long phosphorescence of $\mathrm{SrAl}_{2} \mathrm{O}_{4}: \mathrm{Eu}^{2+}, \mathrm{Dy}^{3+}$ and $\mathrm{CaAl}_{2} \mathrm{O}_{4}: \mathrm{Eu}^{2+}, \mathrm{Nd}^{3+}$. J. Lumin. 72-74, 287-289 (1997).

15. Wang, X. X., Zhang, Z. T., Tang, Z. L. \& Lin, Y. H. Characterization and properties of a red and orange $\mathrm{Y}_{2} \mathrm{O}_{2} \mathrm{~S}$-based long afterglow phosphor. Mater. Chem. Phys. 80, 1-5 (2003).

16. le Masne de Chermont, Q. et al. Nanoprobes with near-infrared persistent luminescence for in vivo imaging. Proc. Natl Acad. Sci. USA 104, 9266-9271 (2007).

17. Jia, D., Lewis, L. A. \& Wang, X. J. $\mathrm{Cr}^{3+}$-doped lanthanum gallogermanate phosphors with long persistent IR emission. Electrochem. Solid-State Lett. 13, J32-J34 (2010).

18. Yan, W. et al. Near infrared long-persistent phosphorescence in $\mathrm{La}_{3} \mathrm{Ga}_{5} \mathrm{GeO}_{14}$ $\mathrm{Cr}^{3+}$ phosphor. Opt. Express 18, 20215-20221 (2010).

19. Maldiney, T. et al. Controlling electron trap depth to enhance optical properties of persistent luminescence nanoparticles for in vivo imaging. J. Am. Chem. Soc. 133, 11810-11815 (2011).

20. Abdukayum, A., Chen, J. T., Zhao, Q. \& Yan, X. P. Functional near infraredemitting $\mathrm{Cr}^{3+} / \mathrm{Pr}^{3+}$ co-doped zinc gallogermanate persistent luminescent nanoparticles with superlong afterglow for in vivo targeted bioimaging. J. Am. Chem. Soc. 135, 14125-14133 (2013).
21. Allix, M. et al. Considerable improvement of long-persistent luminescence in germanium and tin substituted $\mathrm{ZnGa}_{2} \mathrm{O}_{4}$. Chem. Mater. 25, 1600-1606 (2013).

22. Liu, F., Liang, Y. J. \& Pan, Z. W. Detection of up-converted persistent luminescence in the near infrared emitted by the $\mathrm{Zn}_{3} \mathrm{Ga}_{2} \mathrm{GeO}_{8}: \mathrm{Cr}^{3+}, \mathrm{Yb}^{3+}, \mathrm{Er}^{3+}$ phosphor. Phys. Rev. Lett. 113, 177401 (2014).

23. Maldiney, T. et al. The in vivo activation of persistent nanophosphors for optical imaging of vascularization, tumours and grafted cells. Nat. Mater. 13, 418-426 (2014).

24. Li, Y. et al. Tailoring of the trap distribution and crystal field in $\mathrm{Cr}^{3+}$-doped nongallate phosphors with near-infrared long-persistence phosphorescence. NPG Asia Mater. 7, e180 (2015).

25. Li, Z. J. et al. Direct aqueous-phase synthesis of sub-10 nm "Luminous Pearls" with enhanced in vivo renewable near-infrared persistent luminescence. J. Am. Chem. Soc. 137, 5304-5307 (2015).

26. Li, Z. J. et al. In vivo repeatedly charging near-infrared-emitting mesoporous $\mathrm{SiO}_{2} / \mathrm{ZnGa}_{2} \mathrm{O}_{4}: \mathrm{Cr}^{3+}$ persistent luminescence nanocomposites. Adv. Sci. 2, 1500001 (2015).

27. Li, Y., Gecevicius, M. \& Qiu, J. R. Long persistent phosphors-from fundamentals to applications. Chem. Soc. Rev. 45, 2090-2136 (2016).

28. Valero, A. et al. Effect of germicidal UVC light on fungi isolated from grapes and raisins. Lett. Appl. Microbiol. 45, 238-243 (2007).

29. Qin, X., Liu, X. W., Huang, W., Bettinelli, M. \& Liu, X. G. Lanthanide-activated phosphors based on $4 \mathrm{f}-5 \mathrm{~d}$ optical transitions: theoretical and experimental aspects. Chem. Rev. 117, 4488-4527 (2017).

30. Yu, N. Y., Liu, F., Li, X. F. \& Pan, Z. W. Near infrared long-persistent phosphorescence in SrAl2O4:Eu ${ }^{2+}, \mathrm{Dy}^{3+}, \mathrm{Er}^{3+}$ phosphors based on persistent energy transfer. Appl. Phys. Lett. 95, 231110 (2009).

31. Tanner, P. A. et al. $4 f-5 d$ transitions of $\mathrm{Pr}^{3+}$ in elpasolite lattices. Phys. Rev. B 67, 115102 (2003).

32. Pawlik, T. \& Spaeth, J. M. Investigation of the $\mathrm{X}$-ray storage phosphors $\mathrm{Cs}_{2} \mathrm{NaYF}_{6}: \mathrm{Pr}^{3+}$ or Ce ${ }^{3+}$. J. Appl. Phys. 82, 4236-4240 (1997).

33. Duan, C. K., Tanner, P. A., Makhov, V. \& Khaidukov, N. Emission and excitation spectra of $\mathrm{Ce}^{3+}$ and $\mathrm{Pr}^{3+}$ ions in hexafluoroelpasolite lattices. J. Phys. Chem. A 115, 8870-8876 (2011).

34. Larson, A. C. \& Von Dreele, R. B. General Structure Analysis System (GSAS). (Los Alamos National Laboratory, Los Alamos, 2000).

35. Yang, H. \& Kim, Y. S. Energy transfer-based spectral properties of Tb-, Pr-, or Sm-codoped YAG:Ce nanocrystalline phosphors. J. Lumin 128, 1570-1576 (2008).

36. Rezende, M. V. D., Montes, P. J. R., Andrade, A. B., Macedo, Z. S. \& Valerio, M. E $G$. Mechanism of $X$-ray excited optical luminescence (XEOL) in europium doped $\mathrm{BaAl}_{2} \mathrm{O}_{4}$ phosphor. Phys. Chem. Chem. Phys. 18, 17646-17654 (2016).

37. Verwey, J. W. M. \& Blasse, G. Ultraviolet luminescence of $\mathrm{Pr}^{3+}$ in a glass. J. Solid State Chem. 80, 152-155 (1989).

38. Johnson, T. A., Rehak, E. A., Sahu, S. P., Ladner, D. A. \& Cates, E. L. Bacteria inactivation via $\mathrm{X}$-ray-induced UVC radioluminescence: toward in situ biofouling prevention in membrane modules. Environ. Sci. Technol. 50, 11912-11921 (2016).

39. Ravel, B. \& Newville, M. ATHENA, ARTEMIS, HEPHAESTUS: data analysis for X-ray absorption spectroscopy using IFEFFIT. J. Synchrotron Radiat. 12, 537-541 (2005).

40. Kresse, G. \& Furthmüller, J. Efficient iterative schemes for ab initio total-energy calculations using a plane-wave basis set. Phys. Rev. B 54, 11169-11186 (1996).

41. Morgan, B. J. \& Watson, G. W. Intrinsic n-type defect formation in $\mathrm{TiO}_{2}$ : a comparison of rutile and anatase from GGA+U calculations. J. Phys. Chem. C 114, 2321-2328 (2010).

42. Park, S. G., Magyari Köpe, B. \& Nishi, Y. Electronic correlation effects in reduced rutile $\mathrm{TiO}_{2}$ within the LDA+U method. Phys. Rev. B 82, 115109 (2010).

43. Hu, Z. P. \& Metiu, H. Choice of $U$ for DFT+U calculations for titanium oxides. J. Phys. Chem. C. 115, 5841-5845 (2011).

44. Guo, S. Q., Wang, Y. Y., Wang, C., Tang, Z. L. \& Zhang, J. Y. Large spin-orbit splitting in the conduction band of halogen $(\mathrm{F}, \mathrm{Cl}, \mathrm{Br}$, and I) doped monolayer $\mathrm{WS}_{2}$ with spin-orbit coupling. Phys. Rev. B 96, 245305 (2017). 